\title{
Urgent implantation of the Berlin Heart Excor biventricular assist device as a total artificial heart in a patient with single ventricle circulation
}

Klaus Valeske, MD, ${ }^{\mathrm{a}}$ Can Yerebakan, MD, ${ }^{\mathrm{a}}$ Matthias Mueller, MD, ${ }^{\mathrm{b}}$ and Hakan Akintuerk, MD, ${ }^{\mathrm{a}}$ Giessen, Germany

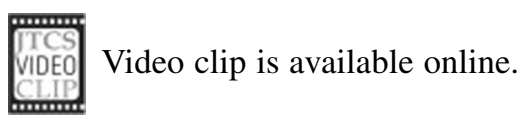

A 19-year-old boy with single ventricle anatomy presented to our department with failing Fontan circulation and secondary end-stage cardiorespiratory failure. $\mathrm{He}$ had been born with hypoplastic left heart syndrome (mitral atresia, aortic stenosis) and had undergone Norwood stage I and II palliations in 1993. Four years later, a total cavopulmonary connection with fenestration was conducted. Since 2005, he had gradually developed signs of cardiac failure, leading to frequent in-hospital

From the Departments of Pediatric Cardiac Surgery ${ }^{a}$ and Pediatric Cardiac

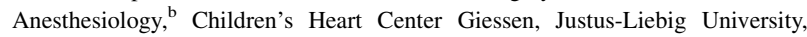
Giessen, Germany.

Disclosures: Authors have nothing to disclose with regard to commercial support.

Drs Valeske and Yerebakan contributed equally to the present report.

Received for publication Sept 12, 2013; revisions received Dec 22, 2013; accepted for publication Jan 10, 2014; available ahead of print Feb 9, 2014.

Address for reprints: Can Yerebakan, MD, Department of Pediatric Cardiac Surgery,

Children's Heart Center Giessen, Justus-Liebig University, Giessen 18057,

Germany (E-mail: can.yerebakan@med.uni-rostock.de).

J Thorac Cardiovasc Surg 2014;147:1712-14

$0022-5223 / \$ 36.00$

Copyright $(2014$ by The American Association for Thoracic Surgery

http://dx.doi.org/10.1016/j.jtcvs.2014.01.012 treatments. Most recently, he had presented to our center with intractable cardiac failure, massive dilatation of the system ventricle, and New York Heart Association class IV. The patient was reported to Eurotransplant as a high-urgency transplantation candidate. Despite highdose inotropic support and maximal therapy for cardiac failure, he developed acute cardiorespiratory failure, with signs of cerebral malperfusion according to nearinfrared spectroscopy (Figure 1). We decided to implant a biventricular assist device.

\section{SURGICAL TECHNIQUE}

It was exceedingly difficult to achieve access to the cardiac structures owing to the massive adhesions in the mediastinum intraoperatively. In addition to the rigidity of the tissue, extensive bleeding ensued owing to the dense adhesions and collateral vessel formation. Also, the patient's cardiorespiratory status deteriorated steadily, leading to a systemic blood pressure of $30 \mathrm{~mm} \mathrm{Hg}$, despite high-dose inotropic support. We gained access to a limited area of the ascending aorta and the superior vena cava, such that urgent cannulation of the patient was established under ongoing cardiorespiratory resuscitation. Extracorporeal circulation was begun with $50 \%$ of the calculated total blood flow until cannulation of the inferior vena cava.

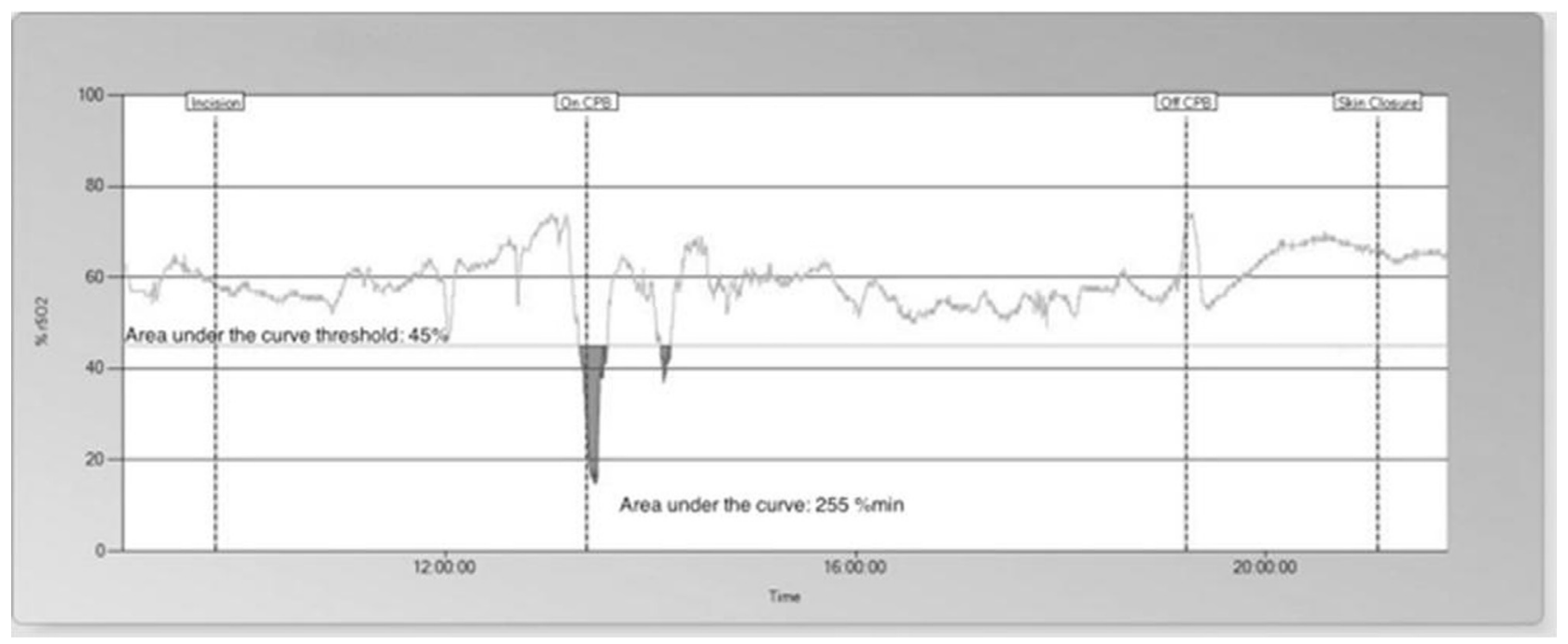

FIGURE 1. Perioperative head near-infrared spectroscopy recording of the patient. 

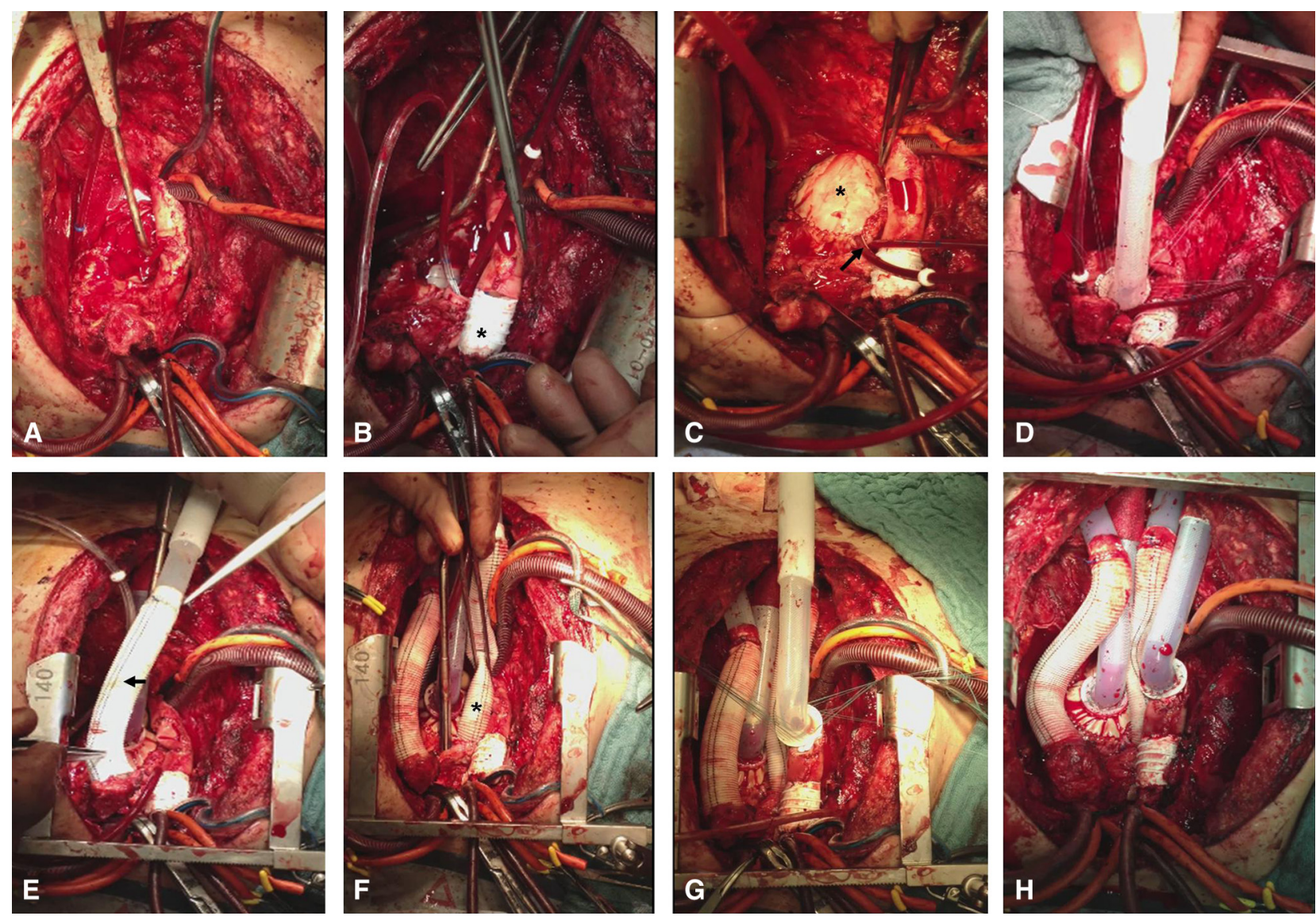

FIGURE 2. A, Situs after explantation of the heart. Note the Fontan pathway and the opened former site of fenestration on the conduit. B, Reestablishment of the continuity between the inferior vena cava and superior vena cava after detachment of the superior vena cava from the pulmonary artery, patch closure of the defect on the pulmonary artery, and Gore-Tex vascular graft (asterisk) interposition between the Fontan conduit and superior vena cava. C, Patch closure of the left atrium with xenograft pericardium (asterisk). Note the vent in the left atrium (arrow). D, Implantation of the Berlin Heart left atrial cannula onto the xenograft pericardial patch. E, Implantation of the Berlin Heart aortic cannula after extension of the cannula using a vascular graft prosthesis (arrow). The aorta of the patient had to be surgically narrowed to adapt to the vascular graft (see Video 1). F, Implantation of the Berlin Heart pulmonary artery cannula (asterisk) after graft extension. G, Implantation of the Berlin Heart right atrial cannula onto the former Fontan conduit at the site of the former fenestration. H, Situs after completion of Berlin Heart biventricular assist device implantation as a total artificial heart. From left to right, aortic, left atrial, pulmonary artery, and right atrial cannulas.

However, even after complete exposure of all cardiac structures, implantation of a biventricular assist device seemed impossible because of massive dilatation of the heart, leading to extremely limited space and brisk bleeding from the collateral vessels in the mediastinum. We decided to explant the heart and implant a Berlin Heart biventricular assist device (Berlin Heart AG, Berlin, Germany) as a total artificial heart. The aorta was divided below the aortic crossclamp, and extensive stepwise preparation around the heart followed. The bidirectional Glenn anastomosis was detached from the right pulmonary artery, and the defect on the right pulmonary artery was closed using xenopericardium. The extracardiac Fontan conduit was removed from the caudal right pulmonary artery, and a new extracardiac conduit was connected between the superior vena cava and the old extracardiac prosthesis. The remainder of the left atrium with the pulmonary veins was reconstructed and closed using a xenopericardial patch ventrally. The fenestration site on the extracardiac conduit was enlarged and reconstructed with xenopericardium. The left atrial cannula of the assist device was connected with a circular opening on the xenopericardium over the left atrium. Two graft-adapted 16F prostheses were connected to the aorta-after downsizing - and to the prosthesis on the former site of the Glenn anastomosis on the pulmonary artery. Finally, the second venous cannula onto the xenopericardium of the former extracardiac conduit was connected. The left atrial and aortic cannulas were connected to an $80-\mathrm{mL}$ chamber, and the right atrial and pulmonary artery cannulas were attached to a smaller $60-\mathrm{mL}$ chamber by considering the bronchial blood flow in a normal circulation. Weaning from the extracorporeal circulation was tolerated well (Figure 2, Video 1). 


\section{POSTOPERATIVE COURSE}

The intensive care course of the patient was complicated, not only by the severely compromised overall preoperative status of the patient, but also by the lacking heart chamber and its reservoir function. Successful extubation of the patient was achieved on the sixth postoperative day. Continuous venovenous hemofiltration was required because of renal failure, which was present preoperatively, and additive renal malperfusion during the phase of preoperative cardiopulmonary resuscitation. The establishment of adequate perfusion to ensure acceptable central venous oxygen saturation revealed a challenge owing to insufficient filling of the left assist chamber. Higher pressures on the left assist system had to be instituted because of clinically relevant hemolysis, the necessity of massive blood transfusion, consecutive hyperbilirubinemia, and cholestasis. Greater central venous pressures, which needed to be kept at 15 to 20 $\mathrm{mm} \mathrm{Hg}$, led to hepatic congestion and ascites later in the treatment course. During the second postoperative week, a severe septic episode ensued and was treated with a broad antibiotic and antimycotic regimen. Mediastinal exploration was performed to remove a massive hematoma formation that was considered a probable source of infection. Subsequently, the infection parameters declined steadily. However, heparinization of the patient led again to mediastinal bleeding with hematoma formation. The patient underwent orthotopic heart transplantation on 23 days after implantation of the Berlin Heart during a phase of maximal medical treatment for the above mentioned problems. We had to accept a relatively smaller donor heart because of the clinical status of the recipient. The pretransplant impairment of renal and liver function was responsible for a longer hospital course after cardiac transplantation. Furthermore, the patient developed severe critical illness polyneuropathy that required a long period of an intensive physiotherapy. He was discharged to a rehabilitation center 7 months after orthotopic heart transplantation, where he achieved excellent clinical improvement within 8 weeks. Therefore, the rehabilitation was classified as a success in that our patient could pursue his normal life with integration of all normal daily activities.

\section{DISCUSSION}

The Berlin Heart Excor assist device has proved its efficacy in infants and children with heart failure in terms of a "bridge-to-transplantation" strategy. ${ }^{1}$ In patients with single ventricle physiology, it has also been used for temporary circulatory assistance, eventually allowing successful heart transplantation. ${ }^{2}$ The treatment of patients presenting with heart failure after total cavopulmonary connection remains an ongoing challenge. The chronic shortage of donor organs frequently hinders timely heart transplantation, such that temporary or long-term mechanical circulatory support could be the last option for these patients. The question of using single or biventricular support for a failing Fontan circulation remains open. In 1 recent report, the use of singlepump Excor support after cardiectomy was described with postoperative hemodynamic challenges similar to those of our patient. Their patient could not be bridged to transplantation. $^{3}$ Novel systems (eg, miniaturized continuous flow pumps) have been considered to possibly improve the outcome of mechanical circulatory support in similar patients. $^{4}$

In our patient, heart failure ensued rapidly, despite maximal medical treatment. Our strategy helped our patient to survive until heart transplantation. To our knowledge, ours is the first report of the implantation of the Berlin Heart Excor biventricular assist device as a total artificial heart enabling a "bridge-to-transplantation" strategy in a patient with single ventricle circulation.

\section{References}

1. Stiller B, Hetzer R, Weng Y, Hummel M, Hennig E, Nagdyman N, et al. Heart transplantation in children after mechanical circulatory support with pulsatile pneumatic assist device. J Heart Lung Transplant. 2003;22:1201-8.

2. Irving CA, Cassidy JV, Kirk RC, Griselli M, Hasan A, Crossland DS. Successful bridge to transplant with the Berlin Heart after cavopulmonary shunt. J Heart Lung Transplant. 2009;28:399-401.

3. VanderPluym CJ, Khoo NS, Rebeyka IM, Buchholz H. Unique case of total artificial cardiac support in failed Fontan circulation after cardiectomy: is continuous flow better than pulsatile flow? J Thorac Cardiovasc Surg. 2013;145:e62-3.

4. Throckmorton AL, Lopez-Isaza S, Downs EA, Chopski SG, Gangemi JJ, Moskowitz W. A viable therapeutic option: mechanical circulatory support of the failing Fontan physiology. Pediatr Cardiol. 2013;34:1357-65. 